\title{
Tranexamic Acid for Blood Loss after Transforaminal Posterior Lumbar Interbody Fusion Surgery: A Double-Blind, Placebo- Controlled, Randomized Study
}

\author{
Bin He $\mathbb{D}^{1},{ }^{1}$ Yuanqiang Li $\mathbb{D}^{1},{ }^{1}$ Shuai Xu $\mathbb{D},{ }^{1}$ Yunsheng Ou $\mathbb{D}^{1},{ }^{1}$ and Jinqiu Zhao $\mathbb{D}^{2}$ \\ ${ }^{1}$ Department of Orthopedics, The First Affiliated Hospital of Chongqing Medical University, Chongqing, China 400016 \\ ${ }^{2}$ Department of Infectious Diseases, The First Affiliated Hospital of Chongqing Medical University, Chongqing, China 400016 \\ Correspondence should be addressed to Yunsheng Ou; ouyunsheng2001@163.com and Jinqiu Zhao; zhaojinqiucy@163.com
}

Received 14 April 2020; Revised 12 July 2020; Accepted 22 July 2020; Published 14 August 2020

Academic Editor: Yukio Hayashi

Copyright ( 2020 Bin He et al. This is an open access article distributed under the Creative Commons Attribution License, which permits unrestricted use, distribution, and reproduction in any medium, provided the original work is properly cited.

\begin{abstract}
Background. Transforaminal lumbar interbody fusion (TLIF) may result in significant blood loss and an increase in blood transfusion. Though tranexamic acid (TXA) is widely studied for the hemostasis of arthroplasty, there is little information on the use of TXA for TLIF surgery. Methods. This prospective randomized, double-blind, placebo-controlled trial was conducted to study the influence of TXA (intravenous bolus of $10 \mathrm{mg} / \mathrm{kg} 15$ minutes before skin incision followed by intravenous infusion of $6-8 \mathrm{mg} / \mathrm{kg} / \mathrm{h}$ up to a total dose of $15 \mathrm{mg} / \mathrm{kg}$ during the surgery) on the blood loss and Enhanced Recovery After Surgery (ERAS) after TLIF surgery. 40 patients were randomized into two groups: TXA group (tranexamic acid) and control group (placebo). Baseline characteristics were comparable between the TXA group and the control group before the surgery. Outcomes assessed included blood loss, total postoperative drainage, time for drainage removal, time to ambulation, hospital stay after surgery, postoperative hemoglobin $(\mathrm{Hb})$ one day after surgery, and adverse events. Results. Compared to patients in the control group after TLIF surgery, patients in the TXA group have significantly reduced intraoperative hemorrhage and time to ambulation after surgery but show similar hospital stay, postoperative drainage, time for drainage removal, postoperative $\mathrm{Hb}$ one day after surgery, and adverse events. Conclusions. TXA shows important ability in controlling blood loss and promoting the ERAS after TLIF surgery.
\end{abstract}

\section{Introduction}

With the increase in aging population, degenerative lumbar diseases (e.g., lumbar disc herniation and spondylolisthesis) have high morbidity [1-3]. Transforaminal lumbar interbody fusion (TLIF) has been widely accepted to treat these diseases and is associated with less trauma and blood loss compared to posterior lumbar interbody fusion [4-6]. However, extensive paravertebral muscle stripping and retraction is still needed to obtain an adequate surgical field during TLIF [7-10].

Because the anatomical structures of the spine have spongy vertebrae with rich blood supply and fragile venous plexus, substantial blood loss frequently occurs during the TLIF and increases postoperative morbidity and prolongs clinical recovery [11-13]. Tranexamic acid (TXA), a syn- thetic lysine analogue of trans-4-aminomethyl-cyclohexane1-carboxylic acid, has been studied to reduce intraoperative blood loss and the need for blood transfusion [14].

Some studies confirmed the efficacy of TXA for controlling blood loss in total knee arthroplasty and total hip arthroplasty $[15,16]$. Only two studies reported the combination use of TXA by preoperative bolus loading and continuous infusion maintenance $[17,18]$. To our knowledge, this prospective randomized, double-blind, placebo-controlled trial is the first study to focus on the safety and effect of TXA combination use (intravenous bolus of $10 \mathrm{mg} / \mathrm{kg} 15$ minutes before skin incision followed by intravenous infusion of 6$8 \mathrm{mg} / \mathrm{kg} / \mathrm{h}$ up to a total dose of $15 \mathrm{mg} / \mathrm{kg}$ during the surgery) on blood loss reduction in TLIF. In particular, ERAS outcomes (e.g., time to ambulation and hospital stay) are evaluated between TXA and placebo. 


\section{Material and Methods}

2.1. Study Design. After obtaining the approval of the Committee of Medical Ethics and the institutional review boards of our institutions, we designed and conducted a randomized, prospective, double-blind, and placebo-controlled trial at our institute from July 2018 to February 2019 following the Consolidated Standards of Reporting Trials (CONSORT) guidelines. Patients or the public were not involved in the design, conduct, reporting, or dissemination plans of our research.

40 patients were randomly allocated into the TXA group (tranexamic acid) or control group (placebo) using computer-based random number generation technique. The TXA group (tranexamic acid) received intravenous bolus of TXA $10 \mathrm{mg} / \mathrm{kg} 15$ minutes before skin incision followed by intravenous infusion of $6-8 \mathrm{mg} / \mathrm{kg} / \mathrm{h}$ up to a total dose of $15 \mathrm{mg} / \mathrm{kg}$ during the surgery. The control group received the equal volume of $0.9 \%$ normal saline as placebo. We obtained the informed written consent from each patient.

$\mathrm{BH}$ was responsible for the randomization of the project and the concealing of TXA by the unified packaging, YQL and SX implemented the intervention of each patient, and JQZ and YSO observed patients and collected the outcome data. The patients, researchers (YQL, SX, JQZ, and YSO), surgeon, and anesthesiologist were blinded to the patients' treatment. At the end of data collection, $\mathrm{BH}$ analyzed the data by groups.

The eligibility criteria were as follows: (1) age between 30 and 80 years old; (2) lumbar disc herniation, stenosis, or spondylolisthesis with unilateral radiculopathy; and (3) one-level or two-level TLIF surgery. The exclusion criteria included lumbar fracture, previous spinal surgery, deformities requiring the correction, coagulation disorder, anticoagulants, or antiplatelet medications. All operations were done by a single senior surgeon. General anesthesia was applied for all patients.

2.2. Surgical Technique. Lumbar interbody fusion surgery was conducted through posterior midline skin incision. Subperiosteal exposure of respective levels was done in the decompression side in order to expose the facet joints. The facet joints in the contralateral side were exposed via separating muscular space between the longissimus and multifidus muscles to expose the facet joint of corresponding levels. Pedicle screws were placed with freehand technique, and connecting rods with an adequate size were installed.

After lamina resection, removal of the corresponding disc, and decompression of nerve root transforaminally, the disc space was distracted to the appropriate height for the insertion of a cage. A cage filled with autogenous bone graft was obliquely inserted into the intervertebral disc space. The pedicle screws and final placement of the cage were confirmed by radiography. After achieving copious irrigation and hemostasis, drainage catheters were placed, and the wounds were closed layer by layer. An intermittent pneumatic compression device was used for the prophylaxis of deep vein thrombosis (DVT) after the surgery.

2.3. Outcome Measures. We recorded the baseline characteristics of each patient and included the age of patients, sex, body mass index (BMI), Hb, hematocrit (HCT), platelet (PLT), prothrombin time (PT), activated partial thromboplastin time (APTT), alanine transaminase (ALT), aspartate transaminase (AST), and surgical time.

Many important outcomes were compared between the two groups, and they involved intraoperative blood loss, total postoperative drainage, time for drainage removal, time to ambulation, hospital stay after surgery, and postoperative $\mathrm{Hb}$ one day after surgery. The drainage removal was conducted when the drainage fluid for $24 \mathrm{~h}$ was less than $30 \mathrm{ml}$. In addition, blood transfusion and adverse events were recorded. Time to ambulation indicated the time period from the end of surgery to the day when patients could walk, while hospital stay after surgery represented the time period from the end of surgery to patient discharge.

2.4. Sample Size. In determining the sample size of the study based on the preliminary experimental results of intraoperative blood loss $(90 \pm 21.1 \mathrm{ml}$ in the TXA group versus $155 \pm$ $79.8 \mathrm{ml}$ in the control group), the power was equal to $80 \%$ and type I error rate was 0.05 . The final sample sizes in each group were 17 or greater, and thus, this study was to recruit 20 patients in each group.

2.5. Statistical Analysis. All parametric data were presented as mean \pm standard deviation (SD). Student's $t$-test for continuous variables or the chi-squared test for dichotomous variables was used to find the significance of study parameters between the TXA group and the control group. These two tests were used to compare the demographic parameters and perioperative and postoperative parameters between the two groups. $P<0.05$ was thought to be statistically significant.

\section{Results}

40 patients were allocated in two groups. There were 15 cases with one-level lumbar interbody fusion and 5 cases with two-level lumbar interbody fusion in each group. Baseline demographic parameters such as age, sex, BMI, Hb, HCT, PLT, PT, APTT, ALT, AST, and surgical time were comparable between the two groups $(P>0.05$, Table 1$)$. The total dose of tranexamic acid was $1631.88 \pm 311.87 \mathrm{mg}$ in the TXA group.

The mean intraoperative blood loss of the TXA group was $91.50 \pm 37.31 \mathrm{ml}$, which was significantly lower than $145 \pm 108.7 \mathrm{ml}$ in the control group $(P=0.04)$. In contrast, there was no statistical significance of postoperative drainage between the TXA group and the control group $(147.7 \pm 70.47$ $\mathrm{ml}$ versus $157.35 \pm 68.3 \mathrm{ml}, P=0.4)$. No significant difference was observed between the two groups in terms of time for drainage removal $(3.25 \pm 0.55$ days versus $3.00 \pm 0.73$ days, $P=0.23$, Table 2 ).

Furthermore, patients in the TXA group required less time to ambulation ( $2.8 \pm 0.52$ days versus $3.35 \pm 0.81$ days, $P=0.049$ ) compared to patients in the control group but showed comparable hospital stay after surgery (5.5 \pm 2.0 days versus $6.8 \pm 1.99$ days, $P=0.54$, Figure 1$)$. No patients in the two groups needed blood transfusion (Table 2). Additionally, 
TABle 1: Demographic data and clinical characteristics.

\begin{tabular}{lccc}
\hline Variable & TXA group & Control group & $P$ value \\
\hline Number & 20 & 20 & \\
Age (year) & $57.95 \pm 12.44$ & $57.9 \pm 11.76$ & 0.99 \\
Male/female & $8 / 12$ & $11 / 9$ & 0.34 \\
BMI $\left(\mathrm{kg} / \mathrm{m}^{2}\right)$ & $25.02 \pm 5.19$ & $24.75 \pm 4.42$ & 0.86 \\
Hb $(\mathrm{g} / \mathrm{l})$ & $137.65 \pm 16.88$ & $131.95 \pm 22.45$ & 0.40 \\
HCT $(\%)$ & $40.86 \pm 4.41$ & $39.24 \pm 5.88$ & 0.33 \\
PLT $\left(10^{9} / \mathrm{l}\right)$ & $183.85 \pm 54.68$ & $174.35 \pm 53.87$ & 0.58 \\
PT $(\mathrm{s})$ & $12.92 \pm 2.2$ & $12.95 \pm 0.70$ & 0.95 \\
APTT (s) & $34.26 \pm 4.28$ & $36.45 \pm 3.31$ & 0.08 \\
ALT (U/l) & $30.65 \pm 38.31$ & $24.45 \pm 19.02$ & 0.52 \\
AST (U/l) & $22.55 \pm 14.02$ & $24.5 \pm 15.62$ & 0.68 \\
Surgical time (min) & $159.20 \pm 29.91$ & $143.65 \pm 36.83$ & 0.15 \\
\hline
\end{tabular}

TXA: tranexamic acid; BMI: body mass index; Hb: hemoglobin; HCT: hematocrit; PLT: platelet; PT: prothrombin time; APTT: activated partial thromboplastin time; ALT: alanine transaminase; AST: aspartate transaminase.

the postoperative $\mathrm{Hb}$ one day after surgery in the TXA group was similar to that in the control group $(122.6 \pm 19.3 \mathrm{~g} / \mathrm{l}$ versus $117.75 \pm 19.03 \mathrm{~g} / \mathrm{l}, P=0.43$, Figure 2 ).

Adverse events were found in 9 patients ( 5 cases in the TXA group, 4 cases in the control group). Superficial wound infection was observed in one patient of each group, and these two patients obtained wound healing after debridement and suturing under local anesthesia. Two patients suffered from hypoproteinemia (one case in each group). In addition, liver dysfunction was found in 3 patients of the TXA group and 2 patients in the control group. These patients all achieved recovery after drug therapy. No DVT was found. The total complications were similar in the two groups $(P=0.71$, Table 2$)$.

\section{Discussion}

The procedures of lumbar spinal fusion surgery include the decompression, instrumentation, correction, and fusion. Intraoperative blood loss during lumbar fusion surgery is estimated to be over $800 \mathrm{ml}$ (range 100 3100 ml) for noninstrumented fusion and $1517 \mathrm{ml}$ (range $360 \sim 7000 \mathrm{ml}$ ) for instrumented fusion [19]. Blood transfusion is occasionally required to treat symptomatic anemia and promote postoperative rehabilitation [20]. Adequate hemostasis can reduce the risk of epidural hematoma formation, which may cause neural compression and neurological deficits [21].

Many methods have been developed to control bleeding during spinal surgery and mainly include minimal invasion procedures, patient positioning, deliberate hypotension, intraabdominal pressure control, infiltration of paraspinal tissues using vasoconstrictors, and pharmacological agents to enhance coagulation [10,22-25]. TXA is known as an antifibrinolytic agent and acts through blocking the interaction of plasminogen and plasmin by competing with the lysine residues on the surface of fibrin to inhibit the fibrinolysis, resulting in clot stabilization [26-29]. Our study suggests that preoperative and intraoperative intravenous infusion of TXA can substantially reduce intraoperative blood loss and time to ambulation but shows no obvious impact on hospital stay after surgery, postoperative drainage, time for drainage removal, or postoperative $\mathrm{Hb}$.

Only two RCTs reported the impact of preoperative and intraoperative intravenous infusion of TXA on blood loss for spinal fusion surgery $[17,18]$. Intravenous tranexamic acid might have the better ability to reduce blood loss than its local infiltration [26]. Low dose $(5 \mathrm{mg} / \mathrm{kg}$ of bolus loading dose and $1 \mathrm{mg} / \mathrm{kg}$ of continuous infusion until $5 \mathrm{~h}$ after surgery) and high dose $(10 \mathrm{mg} / \mathrm{kg}$ of bolus loading dose and $2 \mathrm{mg} / \mathrm{kg}$ of continuous infusion until $5 \mathrm{~h}$ after surgery) of TXA were applied for single-level posterior lumbar interbody fusion, and 24 cases were included in each group. The results found that TXA resulted in the significant decrease in intraoperative blood loss $(385 \pm 139 \mathrm{ml}$ versus $542 \pm 333 \mathrm{ml}$ in the control group, $P=0.03$ ), but there was no statistical difference between the low dose of TXA and the control group $(508 \pm 269 \mathrm{ml}$ versus $542 \pm 333 \mathrm{ml}, P=$ 0.74 ), indicating that high dose of TXA was effective to reduce blood loss for posterior lumbar interbody fusion [18]. Considering the outcomes including $\mathrm{Hb}$ and HCT, high dose of TXA and control intervention resulted in similar change of $\mathrm{Hb}(1.3 \pm 0.6$ versus $1.7 \pm 0.2 \mathrm{~g} / \mathrm{dl}$, respectively, $P=0.75)$ and HCT $(2.3 \pm 1.6$ versus $5.8 \pm 2.3 \%$, respectively, $P=0.15$ ) [18], which was also confirmed by another study involving 50 patients in the TXA group and 46 patients in the control group for posterior lumbar surgery [17].

This RCT investigates the influence of higher dose $(10 \mathrm{mg} / \mathrm{kg} 15$ minutes before skin incision followed by intravenous infusion of $6-8 \mathrm{mg} / \mathrm{kg} / \mathrm{h}$ up to a total dose of $15 \mathrm{mg} / \mathrm{kg}$ during the surgery) of TXA for TLIF than the study conducted by Kim et al. [18]. Theoretically, the better efficacy to reduce blood loss should be observed in our results, but the mean intraoperative blood loss of the two groups was $91.50 \pm 37.31 \mathrm{ml}$ for TXA and $145 \pm 108.7 \mathrm{ml}$ for placebo, respectively. The $P$ value between groups was only equal to 0.04 . These may be caused by the small patient sample and the difference in blood loss due to various levels of surgical trauma. Additionally, no statistical difference in postoperative $\mathrm{Hb}$ remained between the two groups in our results, which was consistent with previous two studies [17, 18], possibly because blood loss of this surgery was not great. Patients can immediately and partially recover from the blood loss, which could not be obviously presented in postoperative $\mathrm{Hb}$.

Our study revealed no incidence of DVT. The total adverse events were similar between the TXA group and the control group. Our results confirmed the efficacy and safety of this TXA for spinal fusion. In addition, time to ambulation after surgery was remarkably reduced by TXA administration, which promotes ERAS. However, the results of intraoperative blood loss and postoperative $\mathrm{Hb}$ were inconsistent, possibly because the amount of blood loss in TLIF was not very high. Patients had sufficient recovery 
TABLE 2: Comparison of clinical outcomes between the TXA group and the control group.

\begin{tabular}{lccc}
\hline Variable & TXA group & Control group & $P$ value \\
\hline Intraoperative blood loss (ml) & $91.50 \pm 37.31$ & $145 \pm 108.7$ & 0.04 \\
Postoperative drainage (ml) & $147.7 \pm 70.47$ & $157.35 \pm 68.3$ & 0.4 \\
Time for drainage removal (day) & $3.25 \pm 0.55$ & $3.00 \pm 0.73$ & 0.23 \\
Blood transfusion & 0 & 0 & - \\
Adverse events & 5 & 4 & 0.71 \\
\hline
\end{tabular}

TXA: tranexamic acid.

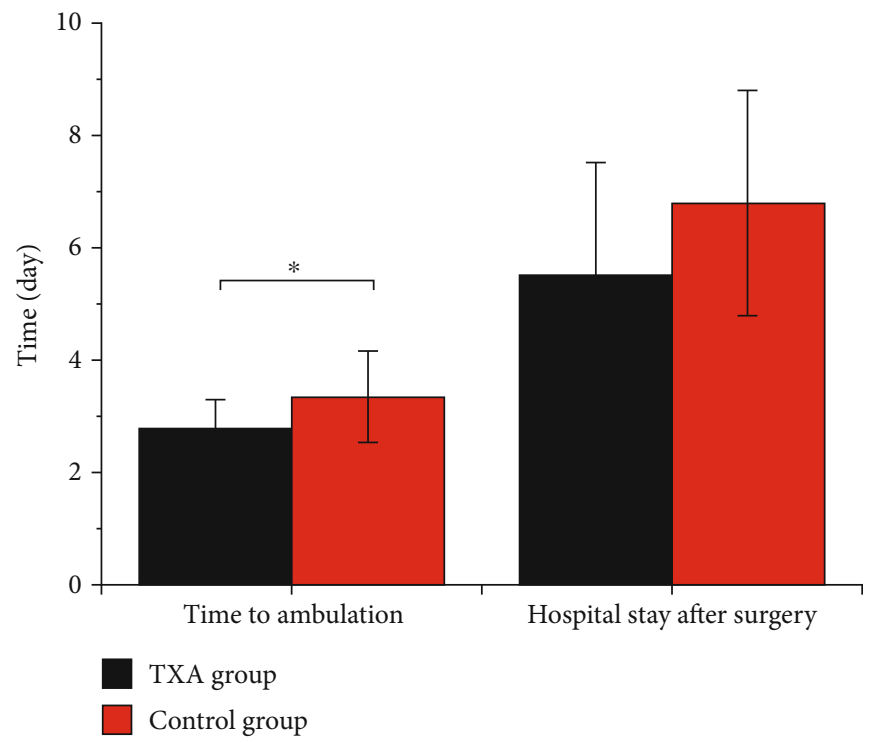

Figure 1: Comparison of time to ambulation and hospital stay after surgery (day) between the two groups. $*$ represents $P<0.05$.



Figure 2: Comparison of preoperative and postoperative hemoglobin ( $\mathrm{Hb}, \mathrm{g} / \mathrm{l})$ one day after surgery between the two groups.

ability after blood loss, and thus, no significant difference of postoperative $\mathrm{Hb}$ was observed between the two groups. The studies regarding complex spinal surgeries (e.g., spinal tuberculosis or deformity correction) with higher amount of blood loss should be conducted to explore the impact of TXA. 


\section{Limitations}

Our study still has several limitations. Firstly, the blood loss in TLIF is relatively low, and future studies should focus on the impact of TXA on complex spinal surgeries with high amount of blood loss. Secondly, the patients in each group have no preoperative or postoperative anemia, and thus, the potential of TXA in reducing blood transfusion cannot be investigated in this study. Thirdly, no DVT is found in the two groups, and we need to explore this dose of TXA on thromboembolism in patients with high risk.

\section{Conclusion}

Intravenous TXA provides additional benefits to blood loss reduction and ERAS in patients with TLIF.

\section{Data Availability}

The datasets generated during and/or analyzed during the current study are available in the ResMan Research Manager repository (http://www.medresman.org.cn/uc/sindex.aspx).

\section{Ethical Approval}

This study was approved by the Committee of Medical Ethics and the institutional review boards of the First Affiliated Hospital of Chongqing Medical University.

\section{Consent}

Informed consent was obtained from all participants.

\section{Disclosure}

All authors approve the publication.

\section{Conflicts of Interest}

The authors declare that there are no conflicts of interest.

\section{Authors' Contributions}

Yuanqiang Li, Jinqiu Zhao, and Bin He conducted the study design, data collection, and statistical analysis. Bin He, Yuanqiang Li, Shuai Xu, Jinqiu Zhao, and Yunsheng Ou conducted the experiment execution. Bin $\mathrm{He}$ and Yunsheng $\mathrm{Ou}$ conducted the data interpretation, manuscript preparation, and literature search. Bin $\mathrm{He}$ and Yunsheng Ou conducted the fund collection. Bin $\mathrm{He}$ and Yuanqiang Li contributed equally to this work.

\section{Acknowledgments}

This study was funded by the Foundation of The First Affiliated Hospital of Chongqing Medical University (PYJJ201813) and Natural Science Foundation of Chongqing (cstc2019jcyj-msxmX0836).

\section{References}

[1] D. Abi-Hanna, J. Kerferd, K. Phan, P. Rao, and R. Mobbs, "Lumbar disk arthroplasty for degenerative disk disease: literature review," World Neurosurgery, vol. 109, pp. 188-196, 2018.

[2] T. Gao, Q. Lai, S. Zhou et al., "Correlation between facet tropism and lumbar degenerative disease: a retrospective analysis," BMC Musculoskeletal Disorders, vol. 18, no. 1, p. 483, 2017.

[3] M. Elsarrag, S. Soldozy, P. Patel et al., "Enhanced recovery after spine surgery: a systematic review," Neurosurgical Focus, vol. 46, no. 4, 2019.

[4] G. Fan, X. Wu, S. Yu et al., "Clinical outcomes of posterior lumbar interbody fusion versus minimally invasive transforaminal lumbar interbody fusion in three-level degenerative lumbar spinal stenosis," BioMed Research International, vol. 2016, Article ID 9540298, 7 pages, 2016.

[5] W. Hu, J. Tang, X. Wu, L. Zhang, and B. Ke, "Minimally invasive versus open transforaminal lumbar fusion: a systematic review of complications," International Orthopaedics, vol. 40, no. 9, pp. 1883-1890, 2016.

[6] K. Eghbal, B. Pourabbas, H. R. Abdollahpour, and R. Mousavi, "Clinical, functional, and radiologic outcome of single- and double-level transforaminal lumbar interbody fusion in patients with low-grade spondylolisthesis," Asian Journal of Neurosurgery, vol. 14, no. 1, pp. 181-187, 2019.

[7] S. A. Salehi, R. Tawk, A. Ganju, F. LaMarca, J. C. Liu, and S. L. Ondra, "Transforaminal lumbar interbody fusion: surgical technique and results in 24 patients," Neurosurgery, vol. 54, no. 2, pp. 368-374, 2004, discussion 374.

[8] B. K. Potter, B. A. Freedman, E. G. Verwiebe, J. M. Hall, D. W. Polly Jr., and T. R. Kuklo, "Transforaminal lumbar interbody fusion," Journal of Spinal Disorders \& Techniques, vol. 18, no. 4, pp. 337-346, 2005.

[9] C. L. Goldstein, F. M. Phillips, and Y. R. Rampersaud, "Comparative effectiveness and economic evaluations of open versus minimally invasive posterior or transforaminal lumbar interbody Fusion," SPINE, vol. 41, p. 1, 2016.

[10] J. Zhao, S. Zhang, X. Li, B. He, Y. Ou, and D. Jiang, "Comparison of minimally invasive and open transforaminal lumbar interbody fusion for lumbar disc herniation: a retrospective cohort study," Medical science monitor : international medical journal of experimental and clinical research, vol. 24, pp. 86938698, 2018.

[11] H. Zhang, Z. X. Chen, Z. M. Sun et al., "Comparison of the total and hidden blood loss in patients undergoing open and minimally invasive transforaminal lumbar interbody fusion," World Neurosurgery, vol. 107, pp. 739-743, 2017.

[12] R. M. Nagabhushan, A. P. Shetty, S. R. Dumpa, B. Subramanian, R. M. Kanna, and R. Shanmuganathan, "Effectiveness and safety of batroxobin, tranexamic acid and a combination in reduction of blood loss in lumbar spinal fusion surgery," Spine, vol. 43, no. 5, pp. E267-E273, 2018.

[13] D. Y. Lee, Y. J. Park, S. Y. Song, S. T. Jeong, and D. H. Kim, "Risk factors for posterior cage migration after lumbar interbody fusion surgery," Asian spine journal, vol. 12, no. 1, pp. 59-68, 2018.

[14] T. Cheriyan, S. P. Maier II, K. Bianco et al., "Efficacy of tranexamic acid on surgical bleeding in spine surgery: a metaanalysis," The Spine Journal, vol. 15, no. 4, pp. 752-761, 2015. 
[15] J. F. DiBlasi, R. P. Smith, J. Garavaglia, J. Quedado, B. M. Frye, and M. J. Dietz, "Comparing cost, efficacy, and safety of intravenous and topical tranexamic acid in total hip and knee arthroplasty," American Journal of Orthopedics, vol. 45, no. 7, pp. E439-E443, 2016.

[16] J. Xie, J. Ma, H. Yao, C. Yue, and F. Pei, "Multiple boluses of intravenous tranexamic acid to reduce hidden blood loss after primary total knee arthroplasty without tourniquet: a randomized clinical trial," The Journal of Arthroplasty, vol. 31, no. 11, pp. 2458-2464, 2016.

[17] H. Shi, Y. Ou, D. Jiang, Z. Quan, Z. Zhao, and Y. Zhu, "Tranexamic acid reduces perioperative blood loss of posterior lumbar surgery for stenosis or spondylolisthesis: a randomized trial," Medicine, vol. 96, no. 1, 2017.

[18] K. T. Kim, C. K. Kim, Y. C. Kim et al., "The effectiveness of low-dose and high-dose tranexamic acid in posterior lumbar interbody fusion: a double-blinded, placebo-controlled randomized study," European spine journal : official publication of the European Spine Society, the European Spinal Deformity Society, and the European Section of the Cervical Spine Research Society, vol. 26, no. 11, pp. 2851-2857, 2017.

[19] H. Moller and R. Hedlund, "Instrumented and noninstrumented posterolateral fusion in adult spondylolisthesis-a prospective randomized study: part 2," Spine, vol. 25, no. 13, pp. 1716$1721,2000$.

[20] E. Y. Tse, W. Y. Cheung, K. F. Ng, and K. D. Luk, "Reducing perioperative blood loss and allogeneic blood transfusion in patients undergoing major spine surgery," The Journal of Bone and Joint Surgery. American Volume, vol. 93, no. 13, pp. 12681277, 2011.

[21] M. J. Sokolowski, T. A. Garvey, J. Perl II et al., "Prospective study of postoperative lumbar epidural hematoma," Spine, vol. 33, no. 1, pp. 108-113, 2008.

[22] D. Cardone and A. A. Klein, "Perioperative blood conservation," European Journal of Anaesthesiology, vol. 26, no. 9, pp. 722-729, 2009.

[23] M. R. Farrokhi, A. P. Kazemi, H. R. Eftekharian, and K. Akbari, "Efficacy of prophylactic low dose of tranexamic acid in spinal fixation surgery: a randomized clinical trial," Journal of Neurosurgical Anesthesiology, vol. 23, no. 4, pp. 290-296, 2011.

[24] F. Janatmakan, S. Nesioonpour, F. Javaherforoosh Zadeh, A. Teimouri, and M. Vaziri, "Comparing the effect of clonidine and dexmedetomidine on intraoperative bleeding in spine surgery," Anesthesiology and pain medicine, vol. 9, no. 1, 2019.

[25] S. M. Frank, J. O. Wasey, I. M. Dwyer, Z. L. Gokaslan, P. M. Ness, and K. M. Kebaish, "Radiofrequency bipolar hemostatic sealer reduces blood loss, transfusion requirements, and cost for patients undergoing multilevel spinal fusion surgery: a case control study," Journal of Orthopaedic Surgery and Research, vol. 9, no. 1, p. 50, 2014.

[26] D. Xu, Q. Zhuang, Z. Li, Z. Ren, X. Chen, and S. Li, “A randomized controlled trial on the effects of collagen sponge and topical tranexamic acid in posterior spinal fusion surgeries," Journal of Orthopaedic Surgery and Research, vol. 12, no. 1, p. 166, 2017.

[27] M. Boin, A. Froehle, M. Lawless, M. Dorweiler, and J. May, "Improved Early Postoperative Range of Motion in Total Knee Arthroplasty Using Tranexamic Acid: A Retrospective Analysis," The Journal of Knee Surgery, vol. 32, no. 2, pp. 160-164, 2019.
[28] L. T. Kuo, W. H. Hsu, C. C. Chi, and J. C. Yoo, "Tranexamic acid in total shoulder arthroplasty and reverse shoulder arthroplasty: a systematic review and meta-analysis," $B M C$ Musculoskeletal Disorders, vol. 19, no. 1, p. 60, 2018.

[29] E. Larson, T. Evans, J. Long, E. Gannon, E. Lyden, and C. Cornett, "Does prophylactic administration of TXA reduce mean operative time and postoperative blood loss in posterior approach lumbar spinal fusion surgery performed for degenerative spinal disease?," Clinical spine surgery, vol. 32 , no. 7 , pp. E353-E358, 2019. 